\title{
Nanostructures imaging via numerical solution of a 3-D inverse scattering problem without the phase information th
}

\author{
Michael V. Klibanov ${ }^{\mathrm{a}}$, Loc H. Nguyen ${ }^{\mathrm{a}}$, Kejia Pan ${ }^{\mathrm{b}, *}$ \\ ${ }^{a}$ Department of Mathematics and Statistics, University of North Carolina at Charlotte, \\ Charlotte, NC 28223, USA \\ ${ }^{b}$ School of Mathematics and Statistics, Central South University, Changsha 410083, \\ China
}

\begin{abstract}
Inverse scattering problems without the phase information arise in imaging of nanostructures, whose sizes are hundreds of nanometers, as well as in imaging of biological cells. The governing equation is the 3-D generalized Helmholtz equation with the unknown coefficient, which represents the spatially distributed dielectric constant. It is assumed in the classical inverse scattering problem that both the modulus and the phase of the complex valued scattered wave field are measured outside of a scatterer. Unlike this, it is assumed here that only the modulus of the complex valued scattered wave field is measured on a certain interval of frequencies. The phase is not measured. In this paper a substantially modified reconstruction procedure of 25] is developed and numerically implemented. Ranges of parameters, which are realistic for imaging of nanostructures, are used in numerical examples. Note that numerical studies were not carried out in [25].
\end{abstract}

Keywords: Phaseless inverse scattering problem, imaging of nanostructures and biological cells, rigorous numerical methods. 2000 MSC: 35R30, 35L05, 78A46.

\footnotetext{
站 The work of first two authors was supported by the Office of Naval Research grant N00014-15-1-2330 and the US Army Research Laboratory and US Army Research Office grant W911NF-15-1-0233.

* Corresponding author

Email addresses: mklibanv@uncc.edu (Michael V. Klibanov), Inguye50@uncc.edu (Loc H. Nguyen), pankejia@hotmail.com (Kejia Pan)
} 


\section{Introduction}

Inverse scattering problems without the phase information occur in imaging of structures whose sizes are of the micron range or less. Recall that 1 micron $(1 \mu \mathrm{m})=10^{-6} \mathrm{~m}$. For example, nano structures typically have sizes of hundreds of nanometers $(\mathrm{nm})$. Either optical of X-ray radiation is used in this imaging. Since $1 \mathrm{~nm}=10^{-9} \mathrm{~m}$, the sizes of these nanostuctures are between $0.1 \mu \mathrm{m}$ and $1 \mu \mathrm{m}$. Therefore, the wavelength $\lambda$ of this imaging should be approximately in the range $\lambda \in[0.05,1] \mu \mathrm{m}$. Another example is in imaging of living biological cells. Sizes of cells are between $5 \mu \mathrm{m}$ and $100 \mu \mathrm{m}$ [37]. It is well known that for the micron range of wavelengths only the intensity of the scattered wave field can be measured, and the phase cannot be measured [12, 13, 18, 36, 41. Here, the intensity is the square modulus of the complex valued wave field.

In a series of recent publications [23, 24, 25, 26] Klibanov and Romanov have analytically developed several reconstruction procedures for determining unknown coefficients of some PDEs from the phaseless scattering data, i.e. in the case when only the modulus of the complex valued wave field is measured outside of scatterers and phase is not measured. In [23, 24] the 3 -D Schrödinger equation was considered and in [25, 26] the 3-D generalized Helmholtz equation was studied.

In our opinion, the main mathematical achievement of publications [25, 26] is that the complicated 3-D inverse scattering problems without the phase information were reduced to the well known Inverse Kinematic Problem (IKP) [28, 38, 39]. It is still unclear how to solve the IKP numerically. Hence, the IKP was linearized in [25, 26]. The latter led to the classical problem of the inversion of the 2-D Radon transform, see, e.g. the book of Natterer [32] for this transform.

Numerical studies were not conducted in [23, 24, 25, 26]. The goal of this publication is to develop and implement a numerical method, which would be based on the idea of the reconstruction procedure of [25]. In [25] the case when the wave field is generated by a point source was considered. However, the numerical method of this paper is significantly different from the procedure of [25]. The main reason of this is that it was assumed in [25] that the frequency $k \in\left(k_{1}, k_{2}\right)$, where $k_{1}>>1$, and, in addition, the interval $\left(k_{1}, k_{2}\right)$ should be sufficiently large in [25]. However, we show in subsection 
4.3 that in the case of the range of parameters, which is realistic for the above mentioned application to imaging of nanostructures, the frequency interval is not as large as it is required in [25. Thus, we develop here a reconstruction procedure, which is substantially different from the one of [25]. We demonstrate computationally that our technique works for that realistic range of parameters.

Recall that, unlike our case, in the standard inverse scattering problem in the frequency domain, one is supposed to determine a scatterer, given measurements of the complex valued wave field outside of that scatterer, see, e.g. [4, 11, 14, 15, 29, 30, 33]. We also refer to the analytical studies of Novikov [34, 35] of two reconstruction procedures for phaseless inverse scattering problems. Although works of Novikov are also concerned with the recovery of unknown coefficients of PDEs, reconstruction procedures of these publications are quite different from the ones of [23, 24, 25, 26]. In addition, works of Ammari, Chow and Zou [2], Bao, Li and Lv [5], Ivanyshyn, Kress and Serranho [17] and Ivanishin and Kress [16] are about numerical reconstructions of surfaces of obstacles from the phaseless data.

As to the uniqueness question for phaseless inverse scattering problems, the first uniqueness theorem was proven by Klibanov and Sacks in the 1-D case [27. Also, see the work of Aktosun and Sacks [1] for a follow up result. Next, uniqueness in the 3-D case was established by Klibanov in [19, 20, 21]. However, proofs in these references are not constructive ones.

In Section 2, we pose the inverse problem which we consider in this paper. In Section 3 we briefly discuss the Lippmann-Schwinger equation, since we use it to generate the data for the inverse problem. In Section 4 we present our reconstruction procedure. In Section 5 we present numerical results. We discuss our results in section 6 .

\section{Problem Settings}

Let $\Omega \subset \mathbb{R}^{3}$ be a bounded domain. Let $G=\{|x|<R\} \subset \mathbb{R}^{3}$ be the ball of the radius $R$ with the center at $\{0\}$. We assume that $\Omega \subset G$. Denote $S(0, R)=\{|x|=R\}$. Let $c(x), x \in \mathbb{R}^{3}$ be a real valued function satisfying the following conditions

$$
\begin{aligned}
c & \in C^{15}\left(\mathbb{R}^{3}\right), \\
c(x) & =1+\beta(x), \\
\beta(x) \geq 0, \beta(x) & =0 \quad \text { for } x \in \mathbb{R}^{3} \backslash \Omega .
\end{aligned}
$$


The smoothness requirement imposed on the function $c(x)$ is clarified in the proof of Theorem 1 of [25]. The non-negativity of the function $\beta(x)$ in (2.3) is due to the fact that the function $c(x) \geq 1$ is the spatially distributed dielectric constant in the above mentioned applications. In other words in the medium of our interest, the dielectric constant exceeds the one of the vacuum. The function $c(x)$ generates the conformal Riemannian metric as

$$
d \tau=\sqrt{c(x)}|d x|,|d x|=\sqrt{\left(d x_{1}\right)^{2}+\left(d x_{2}\right)^{2}+\left(d x_{3}\right)^{2}} .
$$

Here is the assumption which we use everywhere below:

Assumption 1. We assume that geodesic lines of the metric (2.4) satisfy the regularity condition, i.e. for each two points $x, x_{0} \in \mathbb{R}^{3}$, there exists a single geodesic line $\Gamma\left(x, x_{0}\right)$ connecting them.

For $x, x_{0} \in \mathbb{R}^{3}$, let $\tau\left(x, x_{0}\right)$ be the solution of the eikonal equation,

$$
\left|\nabla \tau\left(x, x_{0}\right)\right|^{2}=c(x), \quad \tau\left(x, x_{0}\right)=O\left(\left|x-x_{0}\right|\right), \text { as } x \rightarrow x_{0} .
$$

Here and below all derivatives are with respect to components of the vector $x=\left(x_{1}, x_{2}, x_{3}\right)$. Let $d \sigma$ be the euclidean arc length of the geodesic line $\Gamma\left(x, x_{0}\right)$. Then [39, 40]

$$
\tau\left(x, x_{0}\right)=\int_{\Gamma\left(x, x_{0}\right)} \sqrt{c(\xi)} d \sigma .
$$

Hence, $\tau\left(x, x_{0}\right)$ is the travel time between points $x$ and $x_{0}$ due to the Riemannian metric (2.4). Due to Assumption 1, $\tau\left(x, x_{0}\right)$ is a single-valued function of both points $x$ and $x_{0}$ in $\mathbb{R}^{3} \times \mathbb{R}^{3}$.

Consider the equation

$$
\Delta u+k^{2} c(x) u=0, \quad x \in \mathbb{R}^{3}
$$

where the frequency $k=2 \pi / \lambda$. Here $\lambda>0$ is the wavelength. We now comment on the ranges of parameters used in our computations below. We point out that we work with ranges of parameters which are realistic for the applications to imaging of nanostructures of several hundreds nanometers size as well as of living biological cells. The range of our wavelengths is

$$
\lambda \in[0.078,0.126] \mu m .
$$


Thus, in our computations $\Omega$ is the sphere of the radius $R=1 \mu \mathrm{m}$. We now make variables in (2.7) and (2.8) to be dimensionless. Thus, below all functions, parameters and variables are dimensionless. Introduce a new variable $x^{\prime}=x / 1 \mu m$ while keeping the same notations as above: for brevity. Then elementary steps show that equation (2.7) remains the same. Hence, we obtain that the dimensionless frequency $k=2 \pi / \lambda$ is

$$
k \in[50,80]=\left[k_{1}, k_{2}\right] .
$$

To introduce the point source in equation (2.7), we modify it as

$$
\Delta u+k^{2} c(x) u=-\delta\left(x-x_{0}\right), \quad x \in \mathbb{R}^{3},
$$

where $x_{0} \in \mathbb{R}^{3}$ is the source position. We assume that the function $u\left(x, x_{0}, k\right)$ satisfies the radiation condition,

$$
\frac{\partial u}{\partial r}+i k u=o\left(r^{-1}\right) \text { as } r=\left|x-x_{0}\right| \rightarrow \infty .
$$

Denote $u_{0}\left(x, x_{0}, k\right)$ the solution of the problem 2.10, 2.11) for the case $c(x) \equiv 1$. Then $u_{0}$ is the incident spherical wave,

$$
u_{0}\left(x, x_{0}, k\right)=\frac{\exp \left(-i k\left|x-x_{0}\right|\right)}{4 \pi\left|x-x_{0}\right|} .
$$

Let $u_{s c}\left(x, x_{0}, k\right)$ be the scattered wave, which is due to the presence of scatterers, in which $c(x) \neq 1$. Then

$$
\begin{gathered}
u_{s c}\left(x, x_{0}, k\right)=u\left(x, x_{0}, k\right)-u_{0}\left(x, x_{0}, k\right) \\
=u\left(x, x_{0}, k\right)-\frac{\exp \left(-i k\left|x-x_{0}\right|\right)}{4 \pi\left|x-x_{0}\right|} .
\end{gathered}
$$

It was shown in the book of Colton and Kress 11 that the problem 2.10), (2.11) has unique solution $u$. Moreover, $u$ is in $C^{16+\alpha}\left(\left|x-x_{0}\right| \geq \eta\right)$ for any $\eta>0$ and $\alpha \in(0,1)\left[25\right.$. Here $C^{16+\alpha}$ is the Hölder space.

We model the propagation of the electric wave field in $\mathbb{R}^{3}$ by a single equation 2.10) with the radiation condition 2.11) instead of the full Maxwell's system. This modeling was numerically justified in the work of Beilina [6] in the case of the Cauchy problem for the equation $c(x) v_{t t}=\Delta v$. It was established in [25] that, given Assumption 1, the solution $u\left(x, x_{0}, k\right)$ of the 
problem 2.10, 2.11) is the Fourier transform of the function $v\left(x, x_{0}, t\right)$ with respect to $t$. It was demonstrated in [6] that the component of the electric wave field, which is incident upon the medium, significantly dominates two other components when propagating through the medium. Furthermore, the propagation of that dominating component is well governed by the function $v$. This conclusion was verified via accurate imaging using electromagnetic experimental data in, e.g. Chapter 5 of the book of Beilina and Klibanov [7] and in [9, 10, 44, 45]. In those experimental data only the single component of the scattered electric wave field was measured: the one which was originally incident upon the medium. We believe that the confirmation on the experimental data is meritorious.

In this paper we study the following inverse problem:

Phaseless Inverse Scattering Problem (PISP). Suppose that the following function $f\left(x, x_{0}, k\right)$ is known

$$
f\left(x, x_{0}, k\right)=\left|u_{s c}\left(x, x_{0}, k\right)\right|^{2}, \forall\left(x, x_{0}\right) \in S(0, R) \times S(0, R),
$$

for all $k$ in a certain interval $\left[k_{1}, k_{2}\right] \subset(0, \infty)$. Suppose that the function $c(x)$ satisfies conditions (2.1)-(2.3). Determine the function $\beta(x)$.

\section{Lippmann-Schwinger Equation and the Forward Problem}

To generate the function $f$ in 2.13 for our numerical studies, we need to solve the forward problem (2.10), (2.11). In this section we briefly explain how we solve it via the solution of the Lippmann-Schwinger integral equation. Since in our numerical experiments the function $\beta(x) \neq 0$ only within small inclusions inside of the domain $\Omega$, computations via the Lippmann-Schwinger equation are fast. Recall that by 2.12

$$
u_{\mathrm{sc}}\left(x, x_{0}, k\right)=u\left(x, x_{0}, k\right)-\frac{e^{-i k\left|x-x_{0}\right|}}{4 \pi\left|x-x_{0}\right|}, \quad x \in \mathbb{R}^{3} \backslash\left\{x_{0}\right\} .
$$

It follows from 2.10 and 2.11 that $u_{\mathrm{sc}}$ satisfies

$$
\begin{aligned}
& \Delta u_{\mathrm{sc}}+k^{2} u_{\mathrm{sc}}=-k^{2} \beta u \text { in } \mathbb{R}^{3}, \\
& \frac{\partial u_{\mathrm{sc}}}{\partial r}+i k u_{\mathrm{sc}}=O\left(r^{-1}\right) \text { as } r \rightarrow \infty .
\end{aligned}
$$

Using Theorem 8.3 of the book [11, we derive from (3.1) the LippmannSchwinger equation

$$
u_{\mathrm{sc}}\left(x, x_{0}, k\right)=k^{2} \int_{\Omega} \frac{e^{-i k|x-\xi|}}{4 \pi|x-\xi|} \beta(\xi) u\left(\xi, x_{0}, k\right) d \xi, \quad x \in \mathbb{R}^{3} \backslash\left\{x_{0}\right\} .
$$


Therefore, the integral equation for the total field $u\left(x, x_{0}, k\right)$ is

$$
u\left(x, x_{0}, k\right)=\frac{e^{-i k\left|x-x_{0}\right|}}{4 \pi\left|x-x_{0}\right|}+k^{2} \int_{\Omega} \frac{e^{-i k|x-\xi|}}{4 \pi|x-\xi|} \beta(\xi) u\left(\xi, x_{0}, k\right) d \xi,
$$

for $x \in \mathbb{R}^{3} \backslash\left\{x_{0}\right\}$. Theorems 8.3 and 8.7 of [1] guarantee that there exists unique solution $u \in C^{2}\left(\mathbb{R}^{3} \backslash\left\{x_{0}\right\}\right)$ of the integral equation (3.3). Furthermore, this function $u$ is the solution of problem (2.10), 2.11) and, therefore, it has a higher smoothness (Section 2). In our computations of the forward problem for data generation, we solve equation (3.3) for a discrete set of frequencies $k \in\left[k_{1}, k_{2}\right]$, for $x \in \Omega$ and for $x_{0} \in S(0, R)$. As soon as the function $u\left(x, x_{0}, k\right)$ is found for $x \in \Omega$, its values for points $x \in \mathbb{R}^{3} \backslash \Omega$ can be easily found by substituting these points $x$ in the right hand side of (3.3). This solution produces the function $\left|u_{s c}\left(x, x_{0}, k\right)\right|^{2}=f\left(x, x_{0}, k\right)$ which is the data for our PISP.

\section{The Inverse Problem}

\subsection{The main theorem}

Our reconstruction method is based on Theorem 1. Following [25], let $\zeta=\left(\zeta_{1}, \zeta_{2}, \zeta_{3}\right), \zeta=\zeta\left(x, x_{0}\right)$ be geodesic coordinates of a variable point $x$ with respect to a fixed point $x_{0}$ in the above Riemannian metric (2.4). By Assumption 1, there exists a one-to-one correspondence $x \Leftrightarrow \zeta$ for any fixed point $x_{0}$. Therefore, for any fixed point $x_{0}$ the function $\zeta=\zeta\left(x, x_{0}\right)$ has the inverse function $x=g\left(\zeta, x_{0}\right)$. The function $g$ determines the geodesic line

$$
\Gamma\left(x, x_{0}\right)=\left\{\xi: \xi=g\left(s \zeta_{0}, x_{0}\right), s \in\left[0, \tau\left(x, x_{0}\right)\right]\right\},
$$

where $\zeta_{0}$ is the vector which is tangent to $\Gamma\left(x, x_{0}\right)$ at the point $x_{0}$, is directed towards the point $x$ and also $\left|\zeta_{0}\right|=c^{-1 / 2}\left(x_{0}\right)$.

Consider the Jacobian $J\left(x, x_{0}\right)=\operatorname{det}(\partial \zeta / \partial x)$. By Theorem 1 of [25] $J\left(x, x_{0}\right)>0$ for all $x, x_{0} \in \mathbb{R}^{3}$. Define the function $A\left(x, x_{0}\right)>0$ as

$$
A\left(x, x_{0}\right)=\frac{c\left(x_{0}\right) \sqrt{J\left(x, x_{0}\right)}}{4 \pi \sqrt{c(x)} \tau\left(x, x_{0}\right)} .
$$

Theorem 1 ([25]). Assume that conditions (2.1)- 2.3) and Assumption 1 hold. Fix a point $x_{0} \in \bar{G}$. Then for any point $x \in \bar{G} \backslash\left\{x_{0}\right\}$ the following asymptotic behavior holds

$$
u\left(x, x_{0}, k\right)=A\left(x, x_{0}\right) e^{-i k \tau\left(x, x_{0}\right)}+O\left(\frac{1}{k}\right), k \rightarrow \infty,
$$


where functions $\tau\left(x, x_{0}\right)$ and $A\left(x, x_{0}\right)$ are given in (2.6) and (4.1) respectively.

Combining this theorem with 2.12 , we obtain the following asymptotic behavior of the function $\left|u_{s c}\left(x, x_{0}, k\right)\right|^{2}$ for $x \neq x_{0}$ as $k \rightarrow \infty$

$$
\begin{gathered}
\left|u_{s c}\left(x, x_{0}, k\right)\right|^{2}=A^{2}\left(x, x_{0}\right)+\frac{1}{16 \pi^{2}\left|x-x_{0}\right|^{2}} \\
-\frac{A\left(x, x_{0}\right)}{2 \pi\left|x-x_{0}\right|} \cos \left[k\left(\tau\left(x, x_{0}\right)-\left|x-x_{0}\right|\right)\right]+O\left(\frac{1}{k}\right) .
\end{gathered}
$$

Below in this section, we present our numerical method for solving the above PISP. We point out that since this method ends up with the inversion of the 2-D Radon transform, we are interested in imaging only a 2-D cross-section of the function $\beta(x)$. To do this, it is sufficient to measure the function $f\left(x, x_{0}, k\right)$ for a corresponding 2-D cross-section of the sphere $S(0, R)$, i.e. for $x, x_{0} \in S(0, R) \cap\left\{x_{3}=a\right\}$ for a $a=$ const. $\in(-R, R)$. Without further mentioning we reconstruct images below only in the central 2-D cross-section of the ball $G$, i.e. in the $x_{1}, x_{2}$ plane. We denote this cross-section as $S_{0}(R)=\left\{x: x_{1}^{2}+x_{2}^{2}=R^{2}, x_{3}=0\right\}$. Thus, everywhere below "Radon transform" means the 2 -D Radon transform and $\beta$ means the function $\beta\left(x_{1}, x_{2}, 0\right)$.

Remark 1. Since we are determining the unknown coefficient of equation (2.7), we use in an essential way the dominant term of the asymptotic behavior (4.2). We also refer the reader to [3, 31, 43] for different asymptotic expansion techniques exploited in studying inverse problems with phased scattering data.

\subsection{Radon transform}

In our computations we compute the inverse 2-D Radon transform using a tool box of MATLAB. For every real valued function $g \in L^{2}\left(\mathbb{R}^{2}\right)$, the Radon transform of $g$, suggested by MATLAB, is given by

$$
(\mathcal{R} g)(r, \theta)=\int_{-\infty}^{\infty} g\left(r \cos \theta-x_{2}^{\prime} \sin \theta, r \sin \theta+x_{2}^{\prime} \cos \theta\right) d x_{2}^{\prime},
$$

for $(r, \theta) \in(-\infty, \infty) \times(0, \pi)$. The function above is just the integral along the line $l_{r, \theta}$. Here, $l_{r, \theta}$ is the line obtained by rotating the line $\left\{\left(x_{1}=r, x_{2}\right)\right.$ : 
$\left.x_{2} \in(-\infty, \infty)\right\}$ in the $x_{1} x_{2}$ plane around the origin by the angle $\theta$. For convenience, we name $(r, \theta)$ as the Radon coordinate of the line $l_{r, \theta}$. Let $\mathcal{R}^{-1}$ be the inverse Radon transform.

Consider now our PISP. It is shown below that we can reconstruct the function $\tau\left(x, x_{0}\right)$. Next, the linearization of equation (2.6) implies that we know the following function $f\left(x, x_{0}\right)$ :

$$
f(r, \theta)=\int_{L\left(x, x_{0}\right)} \beta(\xi) d \sigma, \forall\left(x, x_{0}\right) \in S_{0}(R) \times S_{0}(R),
$$

where $(r, \theta)$ is the Radon coordinate of the straight line $L\left(x, x_{0}\right)$ connecting points $x \in S_{0}(R)$ and $x_{0} \in S_{0}(R)$. In (4.4) one should have " $\approx$ " instead of "=" . Thus, (4.4) leads to the following approximate solution of our PISP:

$$
\beta=\mathcal{R}^{-1}(f(r, \theta)), \quad x, x_{0} \in S_{0}(R) .
$$

4.3. Why a large $k$-interval is necessary in the reconstruction procedure of [25]

To explain our need to substantially modify the reconstruction procedure of [25], we briefly explain in this subsection how that procedure reconstructs functions $A\left(x, x_{0}\right)$ and $\tau\left(x, x_{0}\right)$ for $\left(x, x_{0}\right) \in S(0, R) \times S(0, R)$.

Dropping the term $O(1 / k)$ in (4.3) and using (2.13), we obtain for sufficiently large values of $k>>1$ and for $\left(x, x_{0}\right) \in S(0, R) \times S(0, R)$

$$
\begin{gathered}
f\left(x, x_{0}, k\right)=A^{2}\left(x, x_{0}\right)+\frac{1}{16 \pi^{2}\left|x-x_{0}\right|^{2}} \\
-\frac{A\left(x, x_{0}\right)}{2 \pi\left|x-x_{0}\right|} \cos \left[k\left(\tau\left(x, x_{0}\right)-\left|x-x_{0}\right|\right)\right] .
\end{gathered}
$$

Again, as in (4.4) one should have " $\approx$ " instead of "=" in 4.6. Everywhere below $k \geq k_{1}>k_{0}$, where $k_{0}>>1$ is a sufficiently large number. Fix the point $\left(x, x_{0}\right) \in S(0, R) \times S(0, R)$ and consider $f\left(x, x_{0}, k\right)=\varphi(k)$ as the function of $k$ for $k \geq k_{1}$. By 4.6 there exists a number $k_{A} \geq k_{1}$ such that

$$
\begin{aligned}
f^{*}\left(x, x_{0}\right) & =f\left(x, x_{0}, k_{A}\right)=\max _{k \geq k_{1}} f\left(x, x_{0}, k\right) \\
& =\left(A\left(x, x_{0}\right)+\frac{1}{4 \pi\left|x-x_{0}\right|}\right)^{2} .
\end{aligned}
$$


Hence, we find the number $A\left(x, x_{0}\right)$ as

$$
A\left(x, x_{0}\right)=\sqrt{f^{*}\left(x, x_{0}\right)}-\frac{1}{4 \pi\left|x-x_{0}\right|} .
$$

We now find the number $\tau\left(x, x_{0}\right)$. It is possible to figure out whether or not $\tau\left(x, x_{0}\right)=\left|x-x_{0}\right|$. Indeed, it follows from (4.6) that $\tau\left(x, x_{0}\right)=\left|x-x_{0}\right|$ if and only if $\varphi(k)=$ const. for $k \geq k_{1}$. Assume now that $\tau\left(x, x_{0}\right) \neq\left|x-x_{0}\right|$. Then the non-negativity of the function $\beta(x)$ and (2.6) imply that $\tau\left(x, x_{0}\right)>$ $\left|x-x_{0}\right|$. Using (4.6), we obtain that one can choose the number $k_{\tau}>k_{A}$ such that

$$
k_{\tau}=\min \left\{k: k>k_{A}, f\left(x, x_{0}, k\right)=f^{*}\left(x, x_{0}\right)\right\} .
$$

Then 4.6) $(-4.9)$ imply that

$$
k_{\tau}\left(\tau\left(x, x_{0}\right)-\left|x-x_{0}\right|\right)=k_{A}\left(\tau\left(x, x_{0}\right)-\left|x-x_{0}\right|\right)+2 \pi .
$$

Therefore,

$$
\tau\left(x, x_{0}\right)=\left|x-x_{0}\right|+\frac{2 \pi}{k_{\tau}-k_{A}} .
$$

Thus, we need to know the period of the function $\psi(k)$,

$$
\psi(k)=\cos \left[k\left(\tau\left(x, x_{0}\right)-\left|x-x_{0}\right|\right)\right],
$$

in two instances. First, to obtain the number $A\left(x, x_{0}\right)$, using (4.6)-(4.8). Second, to obtain the number $\tau\left(x, x_{0}\right)$ from (4.9)-(4.11). In particular, this means that the smaller the number $\tau\left(x, x_{0}\right)-\left|x-x_{0}\right|$ is, the larger the interval $\left(k_{1}, k_{2}\right)$ must be. However, such large intervals of the frequency $k$ are unavailable in practice, see Figure 1. This has prompted us to develop a different technique, see subsection 4.4.

4.4. Our method of the reconstruction of functions $A\left(x, x_{0}\right)$ and $\tau\left(x, x_{0}\right)$ for $\left(x, x_{0}\right) \in S(0, R) \times S(0, R)$

The method of this subsection is significantly different from the one of subsection 4.3. proposed in [25]. This difference is caused by the fact that [25] is essentially using the assumptions that the frequency interval $k \in\left[k_{1}, k_{2}\right]$ is sufficiently large: see subsection 4.3 and Figure 1. On the other hand, by (2.9) our realistic range of dimensionless frequencies is $\left[k_{1}, k_{2}\right]=[50,80]$ and 


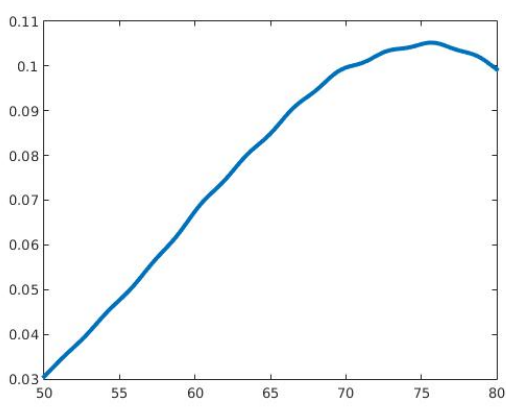

(a) Noiseless data



(b) Data with $5 \%$ noise

Figure 1: A typical behavior of the function $\varphi(k)=f\left(x, x_{0}, k\right)$ for $k \in[50,80]$ as in 2.9$)$. One cannot observe a periodic behavior of $\varphi(k)$ on this interval as required by the method of [25]. Moreover, in the case that the data are noisy, finding $f^{*}\left(x, x_{0}\right)$ and $k_{\tau}$ as in (4.7) and 4.9, respectively, is not easy.

it is obvious from Figure 1 that this interval is not as large as it is required in subsection 4.3. We rewrite (4.6) as

$$
f\left(x, x_{0}, k\right)=a\left(x, x_{0}\right)+b\left(x, x_{0}\right) \cos \left(k \alpha\left(x, x_{0}\right)\right), \quad k \in\left[k_{1}, k_{2}\right]
$$

where

$$
\begin{aligned}
a\left(x, x_{0}\right) & =A^{2}\left(x, x_{0}\right)+\frac{1}{16 \pi^{2}\left|x-x_{0}\right|^{2}} \\
b\left(x, x_{0}\right) & =-\frac{A\left(x, x_{0}\right)}{2 \pi\left|x-x_{0}\right|}, \\
\alpha\left(x, x_{0}\right) & =\tau\left(x, x_{0}\right)-\left|x-x_{0}\right| .
\end{aligned}
$$

We now describe a stable method to extract the unknown quantities $\tau$ and $A$ from $f$. Note that we do not use the differentiation procedure here, since the differentiation is unstable with respect to the noise in the data. Fix an arbitrary pair $\left(x, x_{0}\right) \in S(0, R) \times S(0, R)$. For $k \in\left[k_{1}, k_{2}\right]$, define functions $F_{1}\left(x, x_{0}, k\right), F_{2}\left(x, x_{0}, k\right)$ as

$$
F_{1}\left(x, x_{0}, k\right)=\int_{k_{1}}^{k} f\left(x, x_{0}, \kappa\right) d \kappa, \quad F_{2}\left(x, x_{0}, k\right)=\int_{k_{1}}^{k} F_{1}\left(x, x_{0}, \kappa\right) d \kappa .
$$

For the simplicity of notations, we temporarily ignore the dependence of these functions on $\left(x, x_{0}\right)$. First, we check out whether or not $\alpha=0$. This is 
true if and only if

$$
f(k)=\text { const. for } k \in\left[k_{1}, k_{2}\right] .
$$

On the other hand, in computations of noisy data it is not straightforward to figure out whether or not 4.16 is in place. Thus, we have developed a computational criterion for the verification of $(4.16)$, see $(4.29)$ in this subsection.

Assume now that $\alpha \neq 0$. We obtain

$$
\begin{gathered}
F_{1}(k)=a\left(k-k_{1}\right)+\frac{b}{\alpha} \sin (k \alpha)-\frac{b}{\alpha} \sin \left(k_{1} \alpha\right), \\
F_{2}(k)=a \frac{\left(k-k_{1}\right)^{2}}{2}-\frac{b}{\alpha^{2}} \cos (k \alpha)+\frac{b}{\alpha^{2}} \cos \left(k_{1} \alpha\right)-\frac{b}{\alpha} \sin \left(k_{1} \alpha\right)\left(k-k_{1}\right) .
\end{gathered}
$$

It follows from (4.12), 4.17) and (4.18) that

$$
f(k)+\alpha^{2} F_{2}(k)=\alpha^{2} a \frac{\left(k-k_{1}\right)^{2}}{2}-\alpha b \sin \left(k_{1} \alpha\right)\left(k-k_{1}\right)+a+b \cos \left(k_{1} \alpha\right) .
$$

Note that equation 4.19 is also valid when $\alpha=0$. Consider the vector $\xi$,

$$
\xi=\left(\xi_{1}, \xi_{2}, \xi_{3}, \xi_{4}\right)=\left(\alpha^{2},-\alpha^{2} a, \alpha b \sin \left(k_{1} \alpha\right),-a-b \cos \left(k_{1} \alpha\right)\right)
$$

as an unknown vector. We now should solve equation 4.19) with respect to the vector $\xi$. In (4.19), the parameter $k \in\left[k_{1}, k_{2}\right]$ has infinitely many values. On the other hand, when solving (4.19), we need to find only four numbers $\xi_{1}, \xi_{2}, \xi_{3}, \xi_{4}$. This means that we have an over-determination in the data in (4.19). Hence, to solve equation (4.19), we minimize the functional $J(\xi)$, where

$$
J(\xi)=\frac{1}{2} \int_{k_{1}}^{k_{2}}\left(\xi_{1} F_{2}(k)+\xi_{2} \frac{\left(k-k_{1}\right)^{2}}{2}+\xi_{3}\left(k-k_{1}\right)+\xi_{4}+f(k)\right)^{2} d k .
$$

Since equation (4.19) is linear with respect to the vector $\xi$, then this functional can be minimized via setting to zero its first derivatives with respect to the components of the vector $\xi$, assuming that the resulting matrix is not singular. Hence, a simple calculation yields that the vector $\xi$ solves the linear algebraic system $\mathcal{F} \xi=\mathfrak{f}$ where

$$
\mathcal{F}=\left(\begin{array}{cccc}
\mathcal{F}_{1} & \mathcal{F}_{2} & \mathcal{F}_{3} & \mathcal{F}_{4} \\
\mathcal{F}_{2} & \frac{\left(k_{2}-k_{1}\right)^{5}}{20} & \frac{\left(k_{2}-k_{1}\right)^{4}}{8} & \frac{\left(k_{2}-k_{1}\right)^{3}}{6} \\
\mathcal{F}_{3} & \frac{\left(k_{2}-k_{1}\right)^{4}}{8} & \frac{\left(k_{2}-k_{1}\right)^{3}}{3} & \frac{\left(k_{2}-k_{1}\right)^{2}}{2} \\
\mathcal{F}_{4} & \frac{\left(k_{2}-k_{1}\right)^{3}}{6} & \frac{\left(k_{2}-k_{1}\right)^{2}}{2} & k_{2}-k_{1}
\end{array}\right)
$$


and

$$
\mathfrak{f}=-\left(\begin{array}{c}
\int_{k_{1}}^{k_{2}} f(k) F_{2}(k) d k \\
\int_{k_{1}}^{k_{2}} \frac{\left(k-k_{1}\right)^{2}}{2} f(k) d k \\
\int_{k_{1}}^{k_{2}}\left(k-k_{1}\right) f(k) d k \\
\int_{k_{1}}^{k_{2}} f(k) d k
\end{array}\right) .
$$

Here,

$$
\begin{aligned}
& \mathcal{F}_{1}=\int_{k_{1}}^{k_{2}} F_{2}^{2}(k) d k, \quad \mathcal{F}_{2}=\int_{k_{1}}^{k_{2}} \frac{\left(k-k_{1}\right)^{2}}{2} F_{2}(k) d k \\
& \mathcal{F}_{3}=\int_{k_{1}}^{k_{2}}\left(k-k_{1}\right) F_{2}(k) d k, \quad \mathcal{F}_{4}=\int_{k_{1}}^{k_{2}} F_{2}(k) d k .
\end{aligned}
$$

Remark 2. In practice, the matrix $\mathcal{F}$ might be singular due to the presence of the noise. Sometimes we have observed this computationally when attempting to solve the system $\mathcal{F} \psi=\mathfrak{f}$. We therefore calculate, in Section 5 , the vector $\xi$ by solving

$$
\left(\mathcal{F}^{T} \mathcal{F}+\epsilon \operatorname{Id}\right) \xi=\mathcal{F}^{T} \mathfrak{f}
$$

where $\mathcal{F}^{T}$ is the transpose of $\mathcal{F}$ and $\epsilon>0$ is a sufficiently small number. The term $\epsilon$ Id plays the role of regularization and guarantees that $(4.23)$ is uniquely solvable. In our computations, we set $\epsilon=1$, which is small in comparison to $|\mathcal{F}|_{\max } \geq \frac{\left(k_{2}-k_{1}\right)^{5}}{20}$. Note that $\frac{\left(k_{2}-k_{1}\right)^{5}}{20}$ is the entry on row 2 and column 2 of $\mathcal{F}$. Recall that the vector $\xi=\xi\left(x, x_{0}\right)$ depends on $\left(x, x_{0}\right)$. So, equation 4.23) should be solved for each pair $\left(x, x_{0}\right) \in S(0, R) \times S(0, R)$.

Remark 3. The function

$$
\left(x, x_{0}\right) \in S_{0}(R) \times S_{0}(R) \mapsto \sqrt{\left|\xi_{1}\right|}+\left|x-x_{0}\right|
$$

is the function $\tau\left(x, x_{0}\right)$ and the function $-k \tau\left(x, x_{0}\right)$ approximates the phase of the total wave field $u\left(x, x_{0}, k\right)$ which we need to reconstruct, see (4.2). Having $\tau\left(x, x_{0}\right)$, we solve the quadratic equation (4.3) for $A\left(x, x_{0}\right)$. Note that this quantity might depend on $k$. Hence, we need to average this result with respect to $k$. 
To verify the accuracy of the reconstructed functions $\tau\left(x, x_{0}\right), A\left(x, x_{0}\right)$ for $x, x_{0} \in S_{0}(R)$, we compare them with those of the total field $u\left(x, x_{0}, k\right)$ calculated via the numerical solution of equation (3.3). By (4.2) $A\left(x, x_{0}\right)=$ $\left|u\left(x, x_{0}, k\right)\right|+O(1 / k)$. Since the right hand side of this equality depends on $k$, we assign an approximate value of $A\left(x, x_{0}\right)$ as

$$
A\left(x, x_{0}\right) \approx \frac{1}{k_{2}-k_{1}} \int_{k_{1}}^{k_{2}}\left|u\left(x, x_{0}, k\right)\right| d k .
$$

By (4.2) we have the following approximate formula at $k \gg 1$

$$
P\left(x, x_{0}, k\right)=\int_{k_{1}}^{k} u\left(x, x_{0}, \kappa\right) d \kappa=\frac{u\left(x, x_{0}, k\right)-u\left(x, x_{0}, k_{1}\right)}{-i \tau} .
$$

Multiplying (4.25) by the complex conjugate $\bar{P}\left(x, x_{0}, k\right)$ and integrating the resulting equation with respect to $k$ over the interval $\left(k_{1}, k_{2}\right)$, we obtain

$$
\tau \int_{k_{1}}^{k_{2}}\left|P\left(x, x_{0}, k\right)\right|^{2} d k=\Re \int_{k_{1}}^{k_{2}}\left(i\left(u\left(x, x_{0}, k\right)-u\left(x, x_{0}, k_{1}\right)\right) \bar{P}\left(x, x_{0}, k\right)\right) d k,
$$

which yields an approximation of $\tau\left(x, x_{0}\right)$.

Remark 4. Note that one might find $\tau$ by considering $\Im(\log u)$ and again ignoring the term $O(1 / k)$ in (4.2). However, $|\Im(\log u)| \in[n \pi, n \pi+2 \pi]$ and the integer $n \geq 0$ must be chosen carefully. Therefore, we compare in our figures our calculated values of $A\left(x, x_{0}\right)$ and $\tau\left(x, x_{0}\right)$ with the approximate ones given by 4.24) and 4.26).

Remark 5. Observe that we have approximately found both functions $\tau\left(x, x_{0}\right)$ and $A\left(x, x_{0}\right)$ for $x, x_{0} \in S(0, R)$ without any linearization. However, it is unclear how to find the target function $\beta(x)$ using these functions. Therefore, following [25], we linearize the problem below.

Thus, we have found the function $\tau\left(x, x_{0}\right)$ for all $x, x_{0} \in S(0, R)$. The problem of the determination of the function $\beta(x)$ from the function $\tau\left(x, x_{0}\right)$ given for all $\left(x, x_{0}\right) \in S(0, R) \times S(0, R)$ is called "Inverse Kinematic Problem" [28, 38, 39]. As it was stated in Introduction, it is yet unclear how to solve this problem numerically. Therefore, we solve below the linearized IKP. Assume that $\|\beta\|_{C^{1}(\bar{\Omega})}<<1$. We now linearize the function $\tau\left(x, x_{0}\right)$ with respect to 
the function $\beta$. This linearization can be found in Theorem 11 of Chapter 3 of [28], in $\S$ of Chapter 2 of [38] and in $\S$ of Chapter 3 of [39]. The linearization leads to

$$
\tau\left(x, x_{0}\right)=\left|x-x_{0}\right|+\int_{L\left(x, x_{0}\right)} \beta(\xi) d \sigma .
$$

To be precise, one should have " $\approx$ " instead of " $=$ " in (4.27). Theorem 6 of [25] implies that the accuracy of the linearization (4.27) is proportional to $\|\beta\|_{C^{1}(\bar{\Omega})}^{2}$. Using (4.27), we obtain

$$
\beta=\mathcal{R}^{-1}\left(\tau\left(x, x_{0}\right)-\left|x-x_{0}\right|\right), \quad\left(x, x_{0}\right) \in S_{0}(R) \times S_{0}(R) .
$$

Remark 6. In the case when the straight line $L\left(x, x_{0}\right)$ passes a scatterer, we call $u_{\mathrm{sc}}\left(x, x_{0}, k\right)$ a forward scattering wave. If the line $L\left(x, x_{0}\right)$ does not intersect a scatterer, then $u_{\mathrm{sc}}\left(x, x_{0}, k\right)$ is called either side or back scattering wave. We have observed in our computations that forward scattering waves dominate the side and back ones. We believe that this is the main reason why the inverse Radon transform, which is actually based on an analysis of projections, provides good quality images even though we work with the propagation of waves. Therefore, due to 4.27) and the reconstruction method in 4.28, we find $\tau\left(x, x_{0}\right)$ only for those pairs $\left(x, x_{0}\right) \in S_{0}(R) \times S_{0}(R)$ for which

$$
\frac{\left\|u_{\mathrm{sc}}\left(x, x_{0}, k\right)\right\|_{L^{2}\left(k_{1}, k_{2}\right)}}{\left\|u_{\mathrm{sc}}\left(x, x_{0}, k\right)\right\|_{L^{2}\left(S_{0}(R) \times S_{0}(R) \times\left(k_{1}, k_{2}\right)\right)}} \geq \varepsilon
$$

for a sufficiently small positive number $\varepsilon$ (in our computation $\varepsilon=4 \cdot 10^{-4}$ ). Otherwise, we set $\tau\left(x, x_{0}\right)=\left|x-x_{0}\right|$. This truncation enhances the resolution of the images.

\section{Numerical Results}

We are now in the position to present the numerical results. We first solve the forward problem to collect the data $f\left(x, x_{0}, k\right)=\left|u_{s c}\left(x, x_{0}, k\right)\right|^{2}$, $x, x_{0} \in S_{0}(R), k \in[50,80]$ (see Section 3). Let $\sigma\left(x, x_{0}, k\right)$ be the random variable, which is uniformly distributed in $[-1,1]$. In our computations, we replace $f$ with $f_{\text {noisy }}$, where

$$
f_{\text {noisy }}=\left(1+0.05 \sigma\left(x, x_{0}, k\right)\right) f\left(x, x_{0}, k\right) .
$$

Thus, we have 5\% noise level. The procedure is summarized in Algorithm 1 . 
Algorithm 1 The algorithm to reconstruct $\beta$ from $f_{\text {noisy }}\left(x, x_{0}, k\right),\left(x, x_{0}\right)$ $\in S_{0}(R) \times S_{0}(R), k \in\left[k_{1}, k_{2}\right]$.

1: For each $\left(x, x_{0}\right) \in S_{0}(R) \times S_{0}(R)$, calculate $\alpha\left(x, x_{0}\right)$ as in steps 2-6:

2: If (4.29) holds then,

3: $\quad$ calculate $\mathcal{F}$ and $\mathfrak{f}$ using the noisy data $f_{\text {noisy }}\left(x, x_{0}, k\right)$ (see definitions of $\mathcal{F}$ and $\mathfrak{f}$ in 4.21 and 4.22 ),

4: $\quad$ solve the equation $\mathcal{F} \psi=\mathfrak{f}$ for the vector $\xi\left(x, x_{0}\right)$,

5: $\quad$ set $\alpha\left(x, x_{0}\right)=\sqrt{\left|\xi_{1}\left(x, x_{0}\right)\right|}$.

6: If 4.29 does not hold then, set $\alpha\left(x, x_{0}\right)=0$.

7: For all $\left(x, x_{0}\right) \in S_{0}(R) \times S_{0}(R)$, write $\alpha\left(x, x_{0}\right)=z(r, \theta)$ where $(r, \theta)$ is the Radon coordinate of the line passing through $x$ and $x_{0}$.

8: Calculate the inverse Radon transform of $z(r, \theta)$ to obtain the function $\beta(x)$.

In this section the domain $G=\{x:|x|<1\}$. Hence, in this section $R=1$. The domain $\Omega \subset G$ is the cube inscribed in $G$ and sides of this cube are parallel to coordinate planes. Since by $(2.3)$ the function $\beta(x)=0$ outside of this cube $\Omega$, then images below are presented only in the central $2-\mathrm{D}$ cross section of $\Omega$, i.e. in the square $\left\{\left|x_{1}\right|,\left|x_{2}\right|<\sqrt{2} / 2, x_{3}=0\right\}$. The range of dimensional frequencies $k$ is as in (2.9). Recall that by 2.8 this means that the wavelengths $\lambda \in[0.078,0.126] \mu \mathrm{m}$. Due to the smoothness requirements imposed on the function $\beta$ in Theorems 1 , we introduce the shape function $\varphi \in C_{0}^{\infty}\left(\mathbb{R}^{3}\right)$ to construct some true models based on $\rho$,

$$
\rho(x)= \begin{cases}e^{1-\frac{1}{1-|x|^{2}}} & |x|<1 \\ 0 & \text { otherwise }\end{cases}
$$

Here is how do we construct each inclusion. First, we consider a ball of the radius $r$ with the center at the point $y$. We set the initial function $\beta_{\text {init }}(x)$ as $\beta_{\text {init }}(x)=\gamma$ inside this ball and $\beta_{\text {init }}(x)=0$ outside of it. Here $\gamma=$ const. $>0$. Next, we set $\beta(x)=\beta_{\text {init }}(x) \varphi((x-y) / r)$. Hence, $\gamma$ is the maximal value of the function $\beta(x)$ in that inclusion. If we have two inclusions, we act similarly. Below the "distance between surfaces of two inclusions" means the distance between surfaces of those two original spheres and the "radius" of an inclusion means the radius of that original sphere. Clearly the number $\gamma+1$ can be considered as the inclusion/background contrast of the function $c(x)=1+\beta(x)$. In all our numerical examples 
$\gamma=1$

In this section, we present results of numerical reconstruction by the above algorithm. In particular, we want to evaluate the resolution of our technique. We define the resolution as the minimal distance between surfaces of two inclusions at which they can be separated in our images. Even though distances below are dimensionless, dimensions can be easily assigned: the dimensionless distance $X$ means $X$ microns. Thus, our cases are:

1. Two inclusions of the same radius, which are symmetric with respect to the $x_{2} x_{3}$ plane: see Figures 2,3 and 4 .

2. Two inclusions of the same radii (non-symmetric case): see Figure 5 .

3. Two inclusions with different radii: see Figure 6 .

In each Figure, the square has the center at $\left(x_{1}, x_{2}\right)=(0,0)$ and its side equals $\sqrt{2}$. Hence, this square is inscribed in the circle of the radius $R=1$ with the center at the origin. We display in each figure 2-6:

(a) The 2-D cross-section in the $x_{1}, x_{2}$ plane of the true image.

(b) The Radon transform $(\mathcal{R} \beta)(r, \theta)$ of the true function $\beta\left(x_{1}, x_{2}, 0\right)$.

(c) The reconstructed function $\alpha\left(x, x_{0}\right)=\tau\left(x, x_{0}\right)-\left|x-x_{0}\right|$ for $\left(x, x_{0}\right) \in$ $S_{0}(R) \times S_{0}(R)$ in $(r, \theta)$ coordinates.

(d) The approximate (Remark 4) and reconstructed function $\tau\left(x, x_{0}\right)$ (solid line) when the point $x$ runs along the bottom side of the square and the source is $x_{0}=(1,0,0)$.

(e) The approximate (Remark 4) and reconstructed function $A\left(x, x_{0}\right)$ (solid line) for the same $x, x_{0}$ as in item (d).

(f) The reconstructed image via the solution of PISP by the above Algorithm.

We calculate integrals over the interval $k \in[50,80]$ (Subsection 4.4 ) by their Riemann sums with the step size 0.25 . We have applied a postprocessing procedure to our images. For each vertex $V$ we found a disk $D(V)$ with the center at $V$ of the radius 0.005 . Then we have calculated the average value of the imaged function $\beta$ over all vertices which belong to $\overline{D(V)}$. Next, we 
prescribed that average value as the value of $\beta$ at the vertex $V$. Next, since by (2.3) $\beta \geq 0$, we truncated to zero all negative values of the resulting function $\beta$. Images of Figures 2,6 display functions $\beta$ obtained after this procedure as well as some related information.

It is worth mentioning the software Armadillo by Sanderson [42]. It involves a linear algebra package which is very helpful to speed up our computations.



(a)

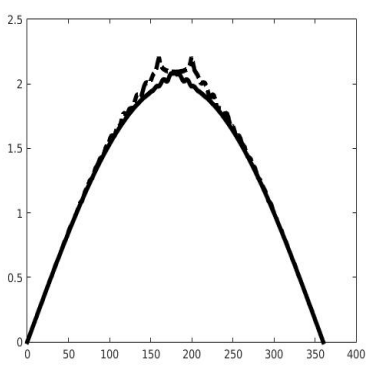

(d)

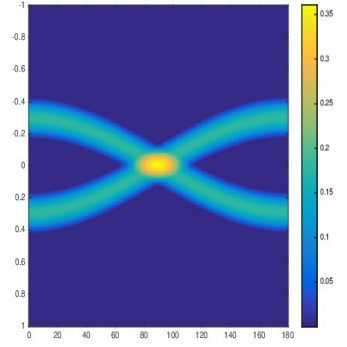

(b)

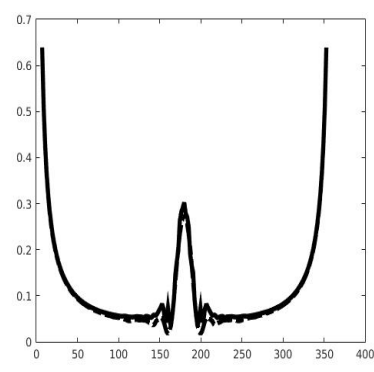

(e)

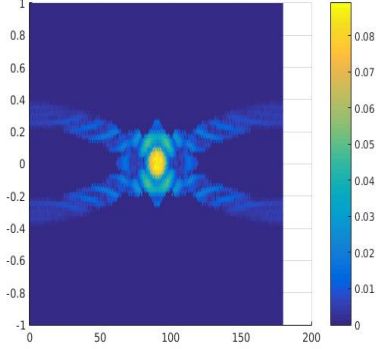

(c)

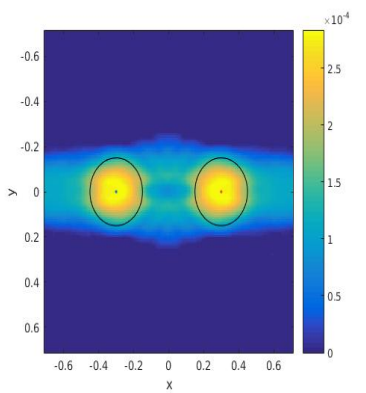

(f)

Figure 2: Two inclusions of the radius 0.15 with the distance 0.3 between their surfaces.

\section{Summary}

In this paper, a substantially modified reconstruction procedure of [25] is applied to solve the 3-D inverse scattering problem without the phase information for the generalized Helmholtz equation (2.10). That modification of the idea of [25] was necessary in order to work with a realistic interval of frequencies $k$. Indeed, the frequency interval required by the procedure of [25] 


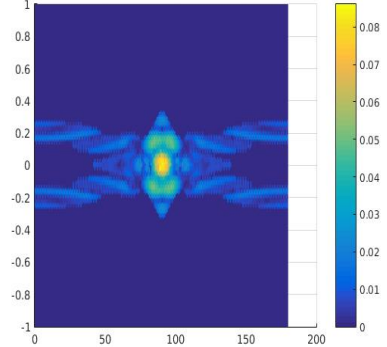

(a)

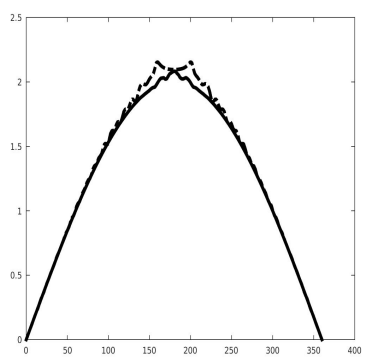

(d)

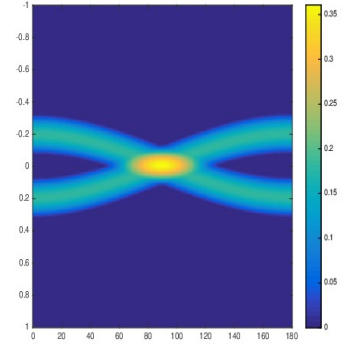

(b)



(e)

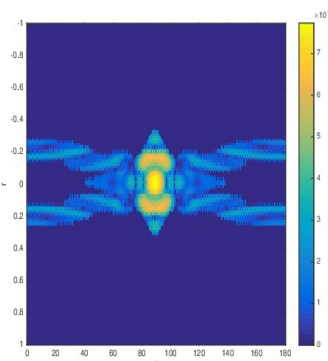

(c)

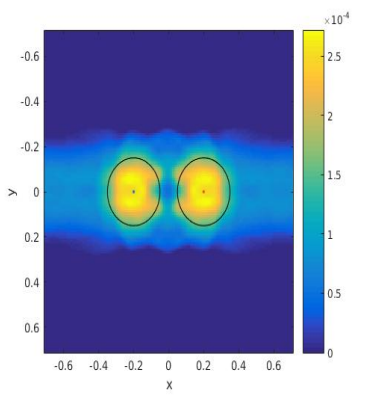

(f)

Figure 3: Two inclusions of the radius 0.15 with the distance 0.1 between their surfaces.

is unreasonably large (subsection 4.3). We have conducted our computations for ranges of parameters, which are realistic for applications to imaging of nanostructures of hundreds of nanometers size. Both X-rays and optical signals on the wavelengths range (2.8) can be used as sources of radiation. Imaging of living biological cells using optical signals is also feasible.

Our procedure, so as the one of [25], reduces the original phaseless inverse scattering problem to the well known Inverse Kinematic Problem. Even though the latter problem has been studied quite intensively in the past [28, 38, 39], it is yet unclear how to solve it numerically. Thus, the last step of both procedures, ours and the one of [25], is the linearization of the Inverse Kinematic Problem. As a result, we end up with the classical problem of the inversion of the Radon transform [32].

By 2.8 the minimal wavelength we use is $\lambda_{\min }=0.078 \mu \mathrm{m}$. On the other hand, we observe on Figure 4 that two inclusions with the distance of $0.025 \mu m=0.32 \lambda_{\min }$ are resolved. Since $0.32 \lambda_{\min }<\lambda_{\min } / 2$, then we observe 


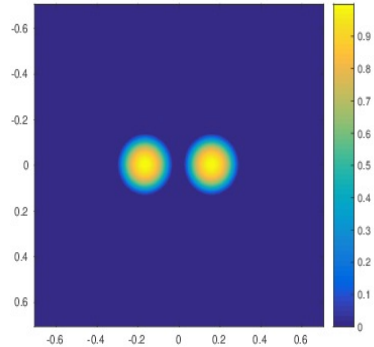

(a)

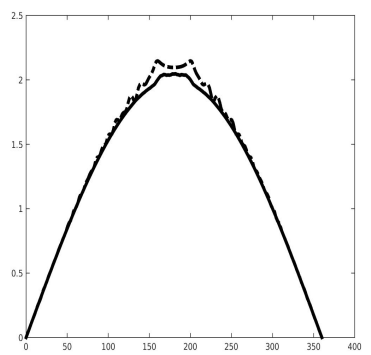

(d)

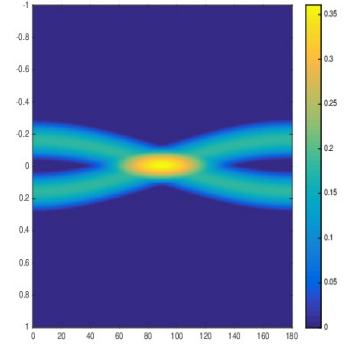

(b)

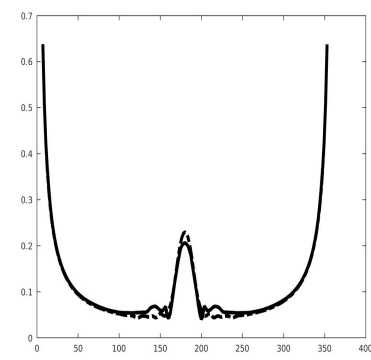

(e)

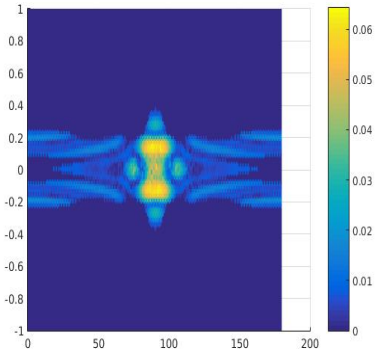

(c)

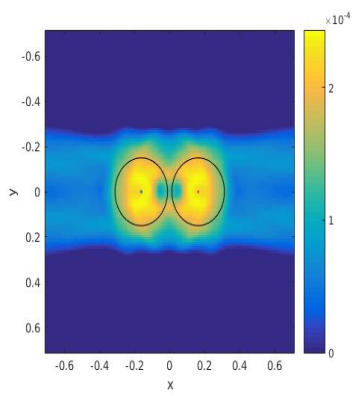

(f)

Figure 4: Two inclusions of the radius 0.15 with the distance 0.025 between their surfaces. This is the case of super resolution, see Section 6 .

a rare case of the super resolution: we differentiate between two inclusions in the case when the distance between them is less than the half of the minimal wavelength. We were unable to obtain a lesser resolution.

Another interesting observation for all our figures 2,6 is that our data are quite far from the range of the operator of the Radon transform: on each of Figures 2, 6 compare (b) and (c). Nevertheless, the application of the inverse Radon transform to our data leads to rather good quality images.

While we image shapes of abnormalities with a good accuracy, the accuracies of the calculated abnormalities/background contrasts are poor. We define the abnormalities/background contrast as

$$
\max _{\text {abnormality }}\left(\frac{1+\beta(x)}{1}\right) .
$$

The correct number in $(6.1)$ is $(1+\gamma) / 1=2$. However our calculated numbers 6.1) vary between 1.0002 and 1.0017. We attribute the latter to 


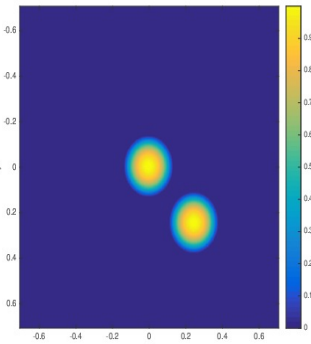

(a)

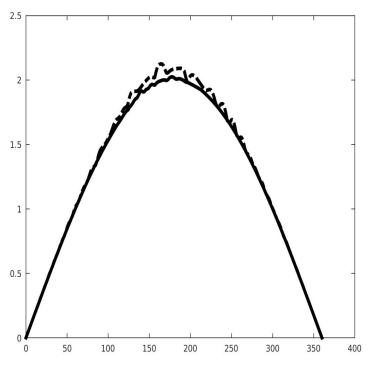

(d)

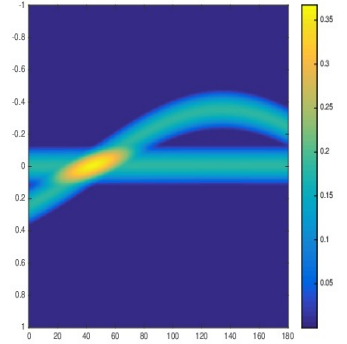

(b)

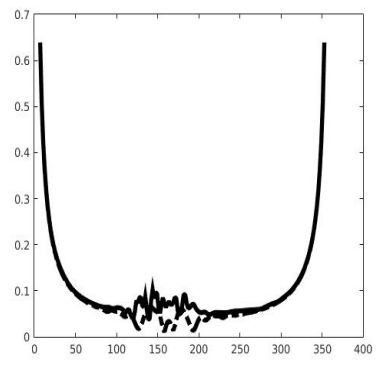

(e)



(c)

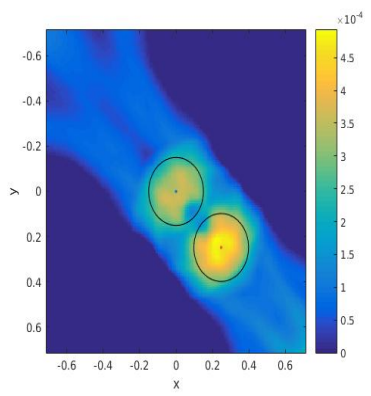

(f)

Figure 5: Two inclusions of the radius 0.15 centered at $(0,0,0)$ and $(0.2475,0.2475,0)$. The distance between their surfaces is 0.05 .

the linearization. On the other hand, Figures 2(d),(e) - 6(d),(e) demonstrate that we accurately reconstruct the function $u\left(x, x_{0}, k\right)$ for $\left(x, x_{0}\right) \in$ $S_{0}(R) \times S_{0}(R)$. Our additional results, which are not shown here, indicate that our method can also accurately reconstruct this function for $\left(x, x_{0}\right) \in$ $S(0, R) \times S(0, R)$, i.e. when points $x$ and $x_{0}$ run over the entire sphere $S(0, R)$ rather than just over the circle $S_{0}(R)$. Hence, to refine the abnormalities/background contrasts $(1+\beta(x)) / 1$, one can use the second stage of the imaging procedure. More precisely, one can apply a modified globally convergent method of [7] for a corresponding Coefficient Inverse Problem. Indeed, it was demonstrated in a number of publications, including detailed studies of experimental data [7, 8, 9, 10, 44, 45, that this method provides accurate values of inclusions/background contrasts.

So, our future works on the topic of this paper will be focused on a twostage numerical procedure. On the first stage we will reconstruct the function 


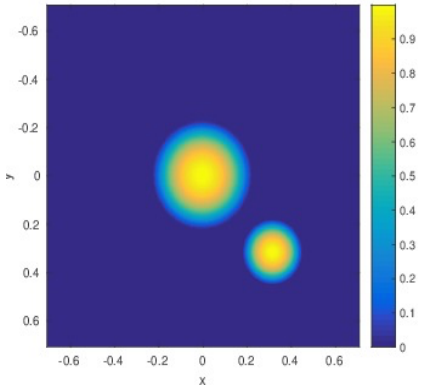

(a)

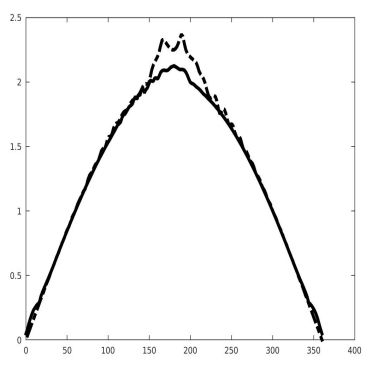

(d)

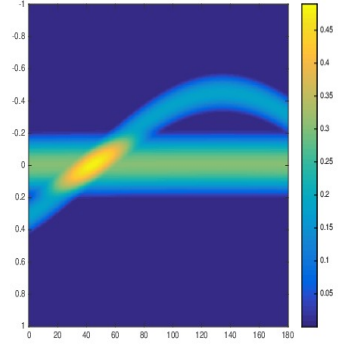

(b)

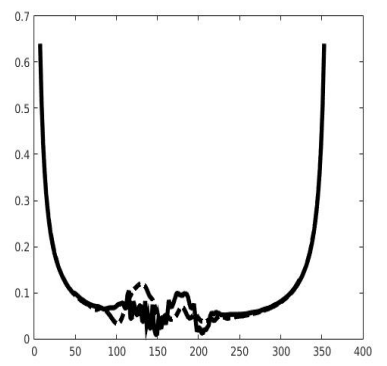

(e)

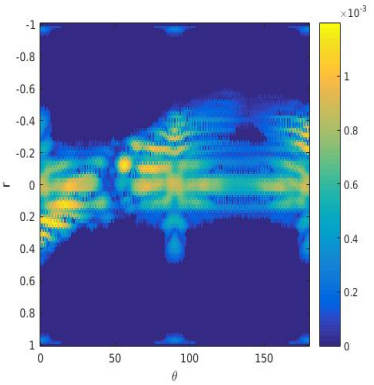

(c)

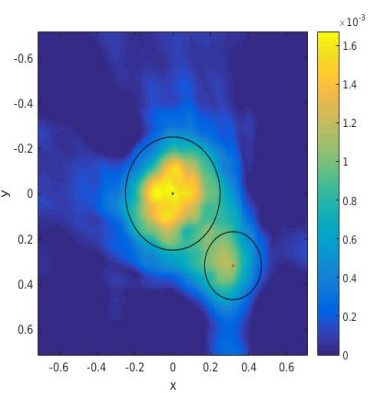

(f)

Figure 6: Two inclusions of the radii 0.25 and 0.15 with the distance 0.05 between their surfaces.

$u\left(x, x_{0}, k\right)$ for $\left(x, x_{0}\right) \in S(0, R) \times S(0, R)$ and for realistic values of $k$. In addition, we will accurately reconstruct locations and shapes of inclusions via applying the inverse Radon transform, as above. Results of this paper indicate that shapes of abnormalities will likely be well reconstructed on this stage. On the second stage, we will use a modified technique of [7] to reconstruct abnormalities/background contrasts $(1+\beta(x)) / 1$, while keeping the shape the same as it was obtained on the first stage.

We note that this is a sort of a reversed two-stage procedure of Chapters 4 and 5 of [7]. In [7] the globally convergent method is applied on the first stage to find locations of abnormalities and abnormalities/background contrasts. On the second stage, a sequence of Tikhonov functionals is minimized in the so-called "adaptivity technique" to find the shapes of inclusions. In doing so, the solution of the first stage is used as the starting point of iterations. Resulting images are quite accurate ones, including the case of experimental 
data, see Chapter 5 in [7] as well as [9, 10]. We also refer to recent publications of $\mathrm{Xu}$ and $\mathrm{Zou}$ [46, 47] for other versions of the adaptivity method for inverse problems. The second option for the globally convergent method is the one of [22], where a globally convex cost functional is constructed using a Carleman Weight Function. In both these globally convergent methods only one source position at a time is used. This means in our case that we can use several sources sequentially to refine the images further.

\section{References}

[1] T. Aktosun, P. E. Sacks, Inverse problem on the line without phase information, Inverse Problems 14 (1998) 211-224.

[2] H. Ammari, Y.T. Chow and J. Zou, Phased and phaseless domain reconstruction in inverse scattering problem via scattering coefficients, SIAM J. Appl. Math. 76 (2016) 1000-1030.

[3] H. Ammari and H. Kang, Reconstruction of Small Inhomogeneities from Boundary Measurements, volume 1846 of Lecture Notes in Mathematics, Springer, 2004.

[4] G. Bao, P. Li, J. Lin, F. Triki, Inverse scattering problems with multifrequencies, Inverse Problems 31 (2015) 093001.

[5] G. Bao, P. Li, J. Lv, Numerical solution of an inverse diffraction grating problem from phaseless data, J. Optical Society of America A 30 (2013) 293-299.

[6] L. Beilina, Energy estimates and numerical verification of the stabilized domain decomposition finite element/finite difference approach for the maxwell's system in time domain, Central European Journal of Mathematics 11 (2013) 702-733.

[7] L. Beilina, M. V. Klibanov, Approximate Global Convergence and Adaptivity for Coefficient Inverse Problems, Springer, New York, 2012.

[8] L. Beilina, N. T. Thành, M. V. Klibanov, M. A. Fiddy, Reconstruction from blind experimental data for an inverse problem for a hyperbolic equation, Inverse Problems 30 (2014) 025002. 
[9] L. Beilina, N.T. Thành, M.V. Klibanov, J.B. Malmberg, Reconstruction of shapes and refractive indices from backscattering experimental data using the adaptivity, Inverse Problems 30 (2014) 105007.

[10] L. Beilina, N.T. Thành, M.V. Klibanov, J.B. Malmberg, Globally convergent and adaptive finite element methods in imaging of buried objects from experimental backscattering radar measurements, J. Computational and Applied Mathematics 290 (2015) 371-391.

[11] D. Colton, R. Kress, Inverse acoustic and electromagnetic scattering theory. second ed., Applied Mathematical Sciences, Springer-Verlag, Berlin, 1998.

[12] A. Darahanau, A. Nikulin, A. Souvorov, Y. Nishino, B. Muddle, T. Ishikawa, Nano-resolution profiling of micro-structures using quantitative x-ray phase retrieval from Fraunhofer diffraction data, Physics Letters A 335 (2005) 494-498.

[13] M. Dierolf, O. Bank, S. Kynde, P. Thibault, I. Johnson, A. Menzel, K. Jefimovs, C. David, O. Marti and F. Pfeiffer, Ptychography \& lenseless x-ray imaging, Europhysics News 39 (2008) 22-24.

[14] G. Hu, J. Li, H. Liu, H. Sun, Inverse elastic scattering for multiscale rigid bodies with a single far-field pattern, SIAM J. Imaging Sciences 7 (2014) 1799-1825.

[15] V. Isakov, Inverse Problems for Partial Differential Equations, Springer, New York, second edition, 2006.

[16] O. Ivanyshyn, R. Kress, Inverse scattering for surface impedance from phase-less far field data, J. Computational Physics 230 (2011).

[17] O. Ivanyshyn, R. Kress and P. Serranho, Huygens' principle and iterative methods in inverse obstacle scattering, Advances in Computational Mathematics 33 (2010) 413-429.

[18] R. Khachaturov, Direct and inverse problems of determining the parameters of multilayer nanostructures from the angular spectrum of the intensity of reflected x-rays, Computational Mathematics and Mathematical Physics 49 (2009) 1781-1788. 
[19] M.V. Klibanov, On the first solution of a long standing problem: Uniqueness of the phaseless quantum inverse scattering problem in 3-d, Applied Mathematics Letters 37 (2014) 82-85.

[20] M.V. Klibanov, Phaseless inverse scattering problems in three dimensions, SIAM J. Appl. Math. 74 (2014) 392-410.

[21] M.V. Klibanov, Uniqueness of two phaseless non-overdetermined inverse acoustics problems in 3-d, Applicable Analysis 93 (2014) 1135-1149.

[22] M. Klibanov, N.T. Thành, Recovering dielectric constants of explosives via a globally strictly convex cost functional, SIAM Journal on Applied Mathematics 75 (2015) 518-537.

[23] M.V. Klibanov, V. G. Romanov, Explicit solution of 3-d inverse scattering problem for the Schrödinger equation: the plane wave case, Eurasian J. of Mathematical and Computer Applications 3 (2015) 48-63.

[24] M.V. Klibanov, V. G. Romanov, The first solution of a long standing problem: Reconstruction formula for a 3-d phaseless inverse scattering problem for the Schrödinger equation, J. Inverse and Ill-Posed Problems 23 (2015) 415-428.

[25] M.V. Klibanov, V. G. Romanov, Reconstruction procedures for two inverse scattering problems without the phase information, SIAM J. Appl.Math 76 (2016) 178-196.

[26] M.V. Klibanov, V. G. Romanov, Two reconsrtuction procedures for a 3-d phaseless inverse scattering problem for the generalized Helmholtz equation, Inverse Problems 32 (2016) 015005.

[27] M.V. Klibabov, P. E. Sacks, Phaseless inverse scattering and the phase problem in optics, J. Math. Physics 33 (1992) 3813-3821.

[28] M. Lavrentiev, V.G. Romanov, V.G. Vasiliev, Multidimensional Inverse Problems for Differential Equations, Springer-Verlag, Berlin, 1970.

[29] J. Li, H. Liu, J. Zou, Locating multiple multiscale acoustic scatterers, SIAM Multiscale Model. Simul. 12 (2014) 927-952.

[30] J. Li, H. Liu, Q. Wang, Enhanced multilevel linear sampling methods for inverse scattering problems, J. Comput. Phys. 257 (2014) 554-571. 
[31] J. J. Liu and M. Sini, On the accuracy of the numerical detection of complex obstacles from far field data using the probe method, SSIAM J. Sci. Comput. 31 (2009) 2665-2687.

[32] F. Natterer, The Mathematics of Computerized Tomography, John Wiley \& Sons, Chichester, 1986.

[33] R.G. Novikov, The inverse scattering problem on a fixed energy level for the two-dimensional Schrödinger operator, J. Functional Analysis 103 (1992) 409-463.

[34] R.G. Novikov, Formulas for phase recovering from phaseless scattering data at fixed frequency, Bulletin des Sciences Mathématiques 26 (2015) 346-359.

[35] R.G. Novikov, Explicit formulas and global uniqueness for phaseless inverse scattering in multidimensions, J. Geometric Analysis 26 (2016) 346-359.

[36] T. Petersena, V. Keastb, D. Paganinc, Quantitative tem-based phase retrieval of mgo nano-cubes using the transport of intensitive equation, Ultramisroscopy 108 (2008) 805-815.

[37] R. Phillips, R. Milo, A feeling for numbers in biology, Proc. Natl. Acad. Sci. USA 106 (2009) 21465-21471.

[38] V.G. Romanov, Integral Geometry and Inverse Problems for Hyperbolic Equations, Springer - Verlag, Berlin, 1974.

[39] V.G. Romanov, Inverse Problems of Mathematical Physics, VNU Science Press, Utrecht, 1987.

[40] V.G. Romanov, Investigation Methods for Inverse Problems, VSP, 2002.

[41] A. Ruhlandt, M. Krenkel, M. Bartels, T. Salditt, Three-dimensional phase retrieval in propagation-based phase-contrast imaging, Physical Review A 89 (2014) 033847.

[42] C. Sanderson, Armadillo: An Open Source C++ Linear Algebra Library for Fast Prototyping and Computationally Intensive Experiments, Technical Report, NICTA, 2010. 
[43] M. Sini and N. T. Thành, Inverse acoustic obstacle scattering problems using multifrequency measurements, Inverse Probl. Imaging 6 (2012) 749-773.

[44] N.T. Thành, L. Bellina, M. V. Klibanov, M. A. Fiddy, Reconstruction of the refractive index from experimental backscattering data using a globally convergent inverse method, SIAM Journal on Scientific Computing 36 (2014) B273-B293.

[45] N.T. Thành, L. Bellina, M. V. Klibanov, M. A. Fiddy, Imaging of buried objects from experimental backscattering time dependent measurements using a globally convergent inverse algorithm, SIAM J. Imaging Sciences 8 (2015) 757-786.

[46] Y. Xu, J. Zou, Analysis of an adaptive finite element method for recovering the Robin coefficient, SIAM J. Control and Optimization 53 (2015) 622-644.

[47] Y. Xu, J. Zou, Convergence of an adaptive finite element method for distributed flux reconstruction, Mathematics of Computation 84 (2015) 2645-2663. 